\title{
Possible role of purinergic signaling in COVID-19
}

\author{
Maria Luiza Mukai Franciosi ${ }^{1}\left[\right.$ ] Millena Daher Medeiros Lima ${ }^{1}\left[\right.$ ] Maria Rosa Chitolina Schetinger ${ }^{2}$. \\ Andréia Machado Cardoso ${ }^{3}$ (1)
}

Received: 19 November 2020 / Accepted: 4 March 2021 / Published online: 19 March 2021

(c) The Author(s), under exclusive licence to Springer Science+Business Media, LLC, part of Springer Nature 2021

\begin{abstract}
The coronavirus disease (COVID-19), caused by SARS-CoV-2 infection, accounts for more than 2.4 million deaths worldwide, making it the main public health problem in 2020. Purinergic signaling is involved in the pathophysiology of several viral infections which makes the purinergic system a potential target of investigation in COVID-19. During viral infections, the ATP release initiates a cascade that activates purinergic receptors. This receptor activation enhances the secretion of proinflammatory cytokines and performs the chemotaxis of macrophages and neutrophils, generating an association between the immune and the purinergic systems. This review was designed to cover the possible functions of purinergic signaling in COVID-19, focusing on the possible role of purinergic receptors such as P2X7 which contributes to cytokine storm and inflammasome NLRP3 activation and P2Y1 that activates the blood coagulation pathway. The possible role of ectonucleotidases, such as CD39 and CD73, which have the function of dephosphorylating ATP in an immunosuppressive component, adenosine, are also covered in detail. Moreover, therapeutic combination or association possibilities targeting purinergic system components are also suggested as a possible useful tool to be tested in future researches, aiming to unveil a novel option to treat COVID-19 patients.
\end{abstract}

Keywords Purinergic Signaling $\cdot$ COVID-19 $\cdot$ Ectonucleotidases $\cdot$ ATP $\cdot$ Adenosine

Andréia Machado Cardoso

andreia.cardoso@uffs.edu.br; deiaa.mc@gmail.com

Maria Luiza Mukai Franciosi

maria.franciosi@estudante.uffs.edu.b;

maria.mukaif@gmail.com

Millena Daher Medeiros Lima

millena.lima@estudante.uffs.edu.br

Maria Rosa Chitolina Schetinger

mariachitolina@gmail.com

1 Medicine Course, Federal University of Fronteira Sul, Campus Chapecó, Chapecó, SC, Brazil

2 Post-Graduation Program in Biological Sciences: Toxicological Biochemistry, CCNE, Federal University of Santa Maria, Santa Maria, RS, Brazil

3 Graduate Program in Biomedical Sciences, Medicine Course, Member of the Brazilian Purine Club, Federal University of Fronteira Sul, Fronteira Sul, Campus Chapecó, Rodovia SC 484 - Km 02, Chapecó, SC 89815-899, Brazil

\section{Introduction}

Since its conception in the early 1970s by Professor Geoffrey Burnstock, the purinergic signaling system has been extensively studied and correlated with several immunological changes, such as those that occur during viral infection and blood clotting $[1,2]$. The COVID-19 pandemic caused by Severe Acute Respiratory Syndrome Coronavirus 2 (SARSCoV-2) started in December 2019 in China and already accounts for more than 2.4 million deaths worldwide by February 24 2021, according to the World Health Organization [3]. The most common symptoms found in clinical presentation are fatigue, myalgia, diarrhea, anorexia, fever, cough, pneumonia, and dyspnea. Although the symptoms are moderate in $80 \%$ of the cases, the disease can worsen in severe acute respiratory syndrome (SARS), defined as a state of bilateral infiltration, hypoxemia, and pulmonary edema $[4,5]$.

According to previous studies on viral infections, there are interconnected changes in the immune system and in the purinergic signaling system, which is composed of purinergic receptors, adenine and guanine nucleosides and 
nucleotides, and ectonucleotidases. Extracellular nucleosides and nucleotides participate in different cellular processes, such as stimulation or inhibition of apoptosis, proliferation, migration, differentiation, secretion of growth factors, and inflammatory mediators [6]. Among the nucleosides receptors, four adenosine receptors (P1) coupled to G protein (A1, A2A, A2B, A3), seven ion channel receptors subtypes for $\mathrm{P} 2 \mathrm{X}$ nucleotides (P2X1-7), and eight G protein-coupled receptors for nucleotides have been identified (P2Y1, 2, 4, 6, 11-14) [7]. P1 and P2 receptors represent important pharmacological targets [8]. Extracellular nucleotides, such as adenosine triphosphate (ATP) and adenosine diphosphate (ADP), are hydrolyzed by ectoenzymes, mainly ecto-nucleoside triphosphate diphosphohydrolase (E-NTPDase or CD39), ecto-nucleotide pyrophosphatase/phosphodiesterase (E-NPP), and ecto-5'-nucleotidase (CD73). This hydrolysis forms adenosine monophosphate (AMP), which will be metabolized by the CD73 enzyme to generate adenosine [7]. Subsequently, adenosine is converted by the enzyme adenosine deaminase (ADA) into inosine and hypoxanthines [9].

In the initial stage of viral infections, the cells of the innate immune system identify the virus and release ATP due to the situation of cellular stress. The ATP is a proinflammatory and chemotactic substance for macrophages and neutrophils that promotes a cascade of purinergic signaling over the following days $[10,11]$. In this way, this review was designed to report the possible pathophysiological mechanisms of COVID-19 related to the purinergic signaling, specially the possible role of some purinergic receptors and ectonucleotidases activities in this scenario. We have also argued to understand how and why enzymes and receptors would be potential pharmacological targets in the treatment of the disease that most generated and still generates worldwide impacts in the last century.

\section{Pathophysiological and immunological concepts of COVID-19}

The infection by SARS-CoV-2 shows important findings. Post-mortem histological findings were observed: alveolar damage with infiltration of $\mathrm{T}$ cells and endothelial presence of thrombi, in addition to angiogenesis about 2.7 times greater compared to patients infected with influenza A, caused by H1N1 $[12,13]$. Other affected organs also had an inflammatory infiltrate of mononucleated cells, such as the heart, kidneys, spleen, and lymph nodes. In laboratory results, there is a decreased lymphocyte count, more pronounced for $\mathrm{CD}^{+} \mathrm{T}$ cells. Besides, there is dysregulation of the blood coagulation pathway due to decreased platelet count, increased degradation of fibrin products, and abnormality in coagulation linked to organ failure in sepsis, which is mainly mediated by inflammatory cytokines
$[4,5,14]$. From an immunological point of view, the entry of SARS-CoV-2 depends on the binding of its spike protein with the cell receptor of the angiotensin-converting enzyme 2 (ACE2) and the transmembrane serine protease 2 (TMPRSS2). The infected epithelial cells produce interferons, which allow a robust innate immune response to occur. In this process, the activated dendritic cells (DCs), macrophages, and neutrophils produce cytokines, which stimulate the adaptive immune response $[15,16]$. Concerning the adaptive immune system, Th $1^{+}$cells secrete granulocyte and macrophage colony-stimulating factor (GM-CSF), and interleukin-6 (IL-6), and monocytes $\mathrm{CD} 14^{+} \mathrm{CD} 16^{+}$, which release IL- 6 and tumor necrosis factor alpha (TNF- $\alpha$ ). This collection of products leads to the infiltration of macrophages, denominated macrophage activation syndrome (MAS), and neutrophils, whose neutrophil extracellular traps (NETs) further increase the release of cytokines, resulting in a cytokine storm, an event associated with COVID-19 gravity and characterized by high activation of immune cells and excessive production of inflammatory cytokines. The NETs are defined as the release of genetic material by the neutrophil, whose purpose is to assist in the innate immune response [17-19].

\section{Purinergic signaling in COVID-19: the possible link}

Connected with the immune response, there are different cascades of purinergic signaling that occur during an infection. In COVID-19, some conditions could stimulate ATP release by infected cells through pannexin 1 channels, such as pathogen invasion (SARS-CoV-2), cell stress, and hypoxia $[10,20]$. If the ATP release happens during COVID19 infection, it is possible that the ATP remains for hours to days in acute response in the extracellular environment exerting its pro-inflammatory effects, until it is metabolized. ATP is a chemoattractant substance for macrophages, and it is recognized as a damage-associated molecular pattern (DAMP) and activates some purinergic receptors (P2X and $\mathrm{P} 2 \mathrm{Y}$ ), which will be described later. Once in the extracellular microenvironment, ATP is dephosphorylated by E-NTPDase (CD39) and ecto-5'-nucleotidase (CD73) (Fig. 1). This enzymatic dephosphorylation process occurs intending to limit the extent and duration of inflammation, since ATP is pro-inflammatory and is degraded in an immunosuppressive product, the adenosine. Throughout the infection, ATP, ADP, AMP, and adenosine perform their functions by binding to purinergic receptors that have affinity. These receptors are present in the membranes of immune cells and platelets and they will be covered in the following topics $[10,11,20]$.

The high concentration of adenosine, resulting from the action of CD73, has immunosuppressive action through the 


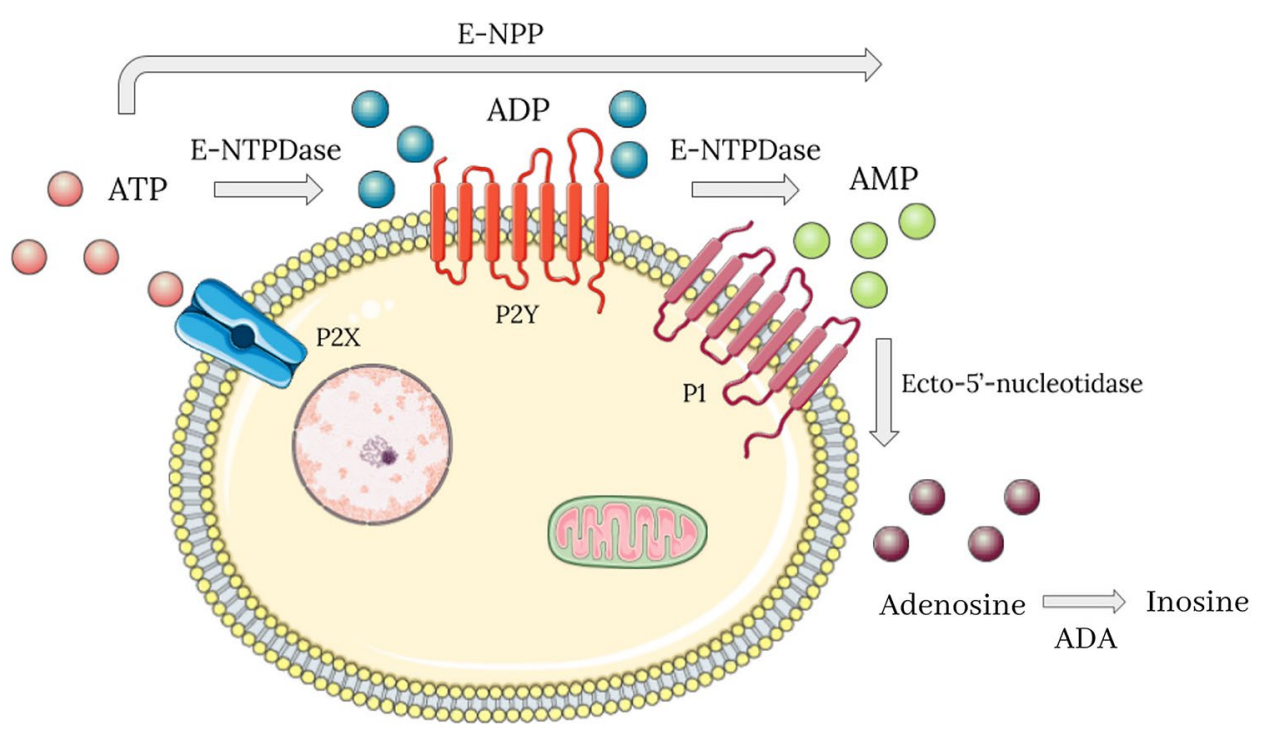

Fig. 1 The purinergic signaling is composed of adenine nucleotides (ATP, ADP, AMP, and adenosine), purinergic receptors, and ectonucleotidases, which are a group of enzymes that dephosphorylate ATP. The purinergic receptors are divided into four $G$ protein-coupled adenosine receptors, designated P1 purinergic receptors, and eighteen P2 purinergic receptors, which mostly have an affinity for ATP. The P2 still are subdivided into four P2X (ionotropic) and eight P2Y ( $\mathrm{G}$ protein-coupled). About the ectonucleotidases, the E-NTPDase

A2A and A2B receptors of infiltrated immune cells [21]. $\mathrm{A} 2 \mathrm{AR}$ is found in most cells and platelets, and promotes anti-inflammatory responses and prevention of platelet aggregation. A2BR is present in macrophages and DCs, it functions being the release of IL- 6 and the vascular endothelial growth factor (VEGF); this receptor is more expressed in inflamed tissues because its activation is dependent on hypoxia [11,22]. Adenosine deaminase (ADA) is responsible for degrading adenosine to inosine, thus increasing levels of this ectonucleotidase may reduce the anti-platelet effect of adenosine, but its absence leads to an inflammatory outcome in the lung in an in vivo study [23, 24].

\section{P2X7R as a potential therapeutic target}

Purinergic receptors are fundamental in understanding the COVID-19, because, in addition to being associated with the immune system, they have also been studied in the context of SARS, fibrosis, and blood clotting [25]. One of the receptors recently discussed in COVID-19 is $\mathrm{P} 2 \mathrm{X} 7$, which is ionotropic, has an affinity for ATP, it is present in T cells, macrophages, neutrophils and DCs, and its function is to activate the cell types previously described and generate an inflammatory environment [11, $20,26]$. When activated by ATP, P2X7R rapidly promotes $\mathrm{K}^{+}$efflux, increased cytosolic $\mathrm{Ca}^{2+}$ concentration, and acts on dephosphorylation of ATP in ADP, and of ADP in AMP. The E-NPP converts ATP into AMP directly. Finally, the ecto-5'nucleotidase turns AMP into adenosine, which is degraded by ADA in inosine. ATP adenosine triphosphate, $A D P$ adenosine diphosphate, $A M P$ adenosine monophosphate, E-NTPDase ecto-nucleoside triphosphate diphosphohydrolase, $E-N P P$ ecto-nucleotide pyrophosphatase/phosphodiesterase, $A D A$ adenosine deaminase

release of cytokines, such as interleukin 1 beta (IL-1 $\beta$ ) in neutrophils, in addition to activating the NLRP3 inflammasome [27]. IL-1 $\beta$ was found at high levels in patients affected by COVID-19 [20, 26, 28]. In general, the outcome of P2X7R signaling is the activation of effector $\mathrm{T}$ cells, $\mathrm{T}$ regulatory cells, natural killer $\mathrm{T}$ cells (NKT), monocytes, macrophages, and DCs. This means that it simultaneously helps the innate and adaptive immune response [20]. Di Virgilio et al. [20] proposed that the use of P2X7R antagonistic substances could contribute to the treatment of COVID-19, since they would reduce the pro-inflammatory effects of ATP released in the initial phase of the infection, which consequently could decrease the activation of macrophages and other cascades of proinflammatory cytokines associated to lung injury caused by SARS-CoV-2. Furthermore, Cicko et al. [29], in a research with patients affected by Acute Respiratory Distress Syndrome (ARDS) and in mice whose lung injury was induced with LPS, found increased ATP levels in the bronchoalveolar fluid, which could be an outcome associated with P2X7R stimulation. This receptor is also a powerful trigger for the production of reactive oxygen species (ROS), which can inhibit lymphocyte functions and lead to sustained cytokine release, factors that would further progress inflammation. The drugs with antagonistic action in P2X7R, especially with a focus on depression, are in Phase II [20]. Therefore, P2X7R can be characterized as 
an important therapeutic potential for COVID-19 and other serious respiratory infections caused by other pathogens [29].

\section{Possible functions performed by P2Y2R and P2Y6R in the immunological context of the infection}

Regarding metabotropic receptors, findings in animal models point to an association between viral pneumonia and $\mathrm{P} 2 \mathrm{Y} 2 \mathrm{R}$. One aspect of $\mathrm{P} 2 \mathrm{Y}$ receptors is its association with $\mathrm{G}$ protein, so its prolonged stimulation can cause desensitization of the receptor [26]. This receptor is expressed in monocytes, DCs, and lymphocytes. The outcome of the $\mathrm{P} 2 \mathrm{Y} 2$ activation is chemotaxis and immune cells activation, which contributes especially to the innate immune response [20]. Vanderstocken et al. (2012) [30] observed that the absence of P2Y2R in mice infected with viruses increases mortality rates, which could be triggered by lower viral clearance leading to more persistent viral load, enhancing the levels of cytokines in bronchoalveolar fluid and promoting a worse state of inflammation. In convergence with COVID-19, which has higher levels of IL-6 in the blood of affected patients, authors also found in this animal model infected by the pneumonia virus of mice the increase of IL- 6 according to the difficulty of establishing and the immunological clearance [15]. Despite this, in this model, ATP levels and the infiltration of macrophages and neutrophils were not increased, which could happen due to the decrease in recruitment of $\mathrm{Th} 1^{+}$cells [30]. There is a need for further studies to clarify the issue of P2Y2R and its effects on cytokines in the context of COVID-19.

The P2Y6 receptor, in turn, was investigated in an animal model in the context of complicated bacterial pneumonia (Pseudomonas aeruginosa) and ventilator-induced lung injury [31]. In patients with COVID-19, there is an inflammatory infiltrate with neutrophils and the application of ventilators is common in severe cases of SARS [5]. In this research conducted by Zheng et al. [31] on P2Y6R, there was an increment in the $\alpha$-defensins released by neutrophils, which can kill microorganisms, but in excess contributes to the inflammatory response with high levels of cytokines (IL-1 $\beta$, IL-6, and TNF- $\alpha$ ) and injury to the epithelium and pulmonary endothelium. The intervention occurred through the use of MRS2578, a selective P2Y6R inhibitor, which attenuated the inflammatory state without affecting the antibacterial properties of $\alpha$-defensins. The P2Y6R block represents, therefore, a possibility of an important therapeutic target in the context of the purinergic system and pneumonia, especially those caused by response to viruses, such as COVID-19. It is necessary to consider that the ventilation, which is a strategy to improve oxygenation, could be a prediction of the bacterial infection [32]. This co-infection of SARS-CoV-2 and another microorganism has the potential to worsen clinical conditions and increase mortality in these patients. There are bacteria involved in ventilator-associated pneumonia: Escherichia coli, Klebsiella pneumonia, Pseudomonas aeruginosa, Acinetobacter baumannii, and Staphylococcus aureus. Most of them are associated with resistance to multidrug resistance [33].

\section{Thrombosis and P2Y12R in COVID-19}

In addition to inflammatory infiltrates, abnormalities in coagulation were observed, such as deposits of intra-alveolar fibrin and disseminated intravascular coagulation in patients diagnosed with COVID-19 [12]. Infected patients also showed convergent results in pathophysiological findings with an increase in D-dimers, which is the product of fibrin degradation, and IL-6, which is being released in excess, contributing to the increase in fibrinogen levels [34, 35]. Findings with hospitalized patients due to COVID-19 showed that $25 \%$ to $31 \%$ developed venous thromboembolism, a percentage referring to research carried out in Wuhan and Europe [36, 37]. The pathophysiology of this dysregulation in the coagulation pathway is not well understood, but in a study with serological markers of neutrophils, an important contribution of NETs to coagulation was observed, considering these "networks" are anti-microbial structures and can collaborate for the propagation of intravascular thrombosis through the activation of factor XII which, in turn, activates platelet functions [38, 39].

Thrombosis has several aspects associated with the purinergic signaling system. Among them, the cascade of dephosphorylation promoted by CD39, which degrades ATP into ADP and AMP, has demonstrated protection against platelet activation in vivo. The importance of this enzymatic action is in the effects of ADP in the P2Y12 receptor, which is metabotropic and is present in platelets. The function of $\mathrm{P} 2 \mathrm{Y} 12$ is monocytic activation and platelet aggregation through the activity of thrombin, collagen, and thromboxane A2 [11, 40, 41]. There are drugs developed that selectively inhibit P2Y12 and that are already commercialized with an indication for some cardiovascular diseases, for example, clopidogrel $[42,43]$. It was recently reported that both clopidogrel and ticagrelor significantly reduced the formation of platelet-monocyte aggregates and peak levels of major proinflammatory cytokines, including TNF $\alpha$ and IL-6, and suppressed D-dimer generation which is elevated in several fatal cases of COVID-19 [44, 45]. However, there is no research and scientific evidence to suggest the effectiveness of clopidogrel and similar drugs for the treatment of thrombosis due to COVID-19. There is a need, therefore, for research to be 
carried out for this purpose, since other anti-coagulants are already being studied, as is the case of heparin [4]

Other receptors related to coagulation dysregulation are $\mathrm{P} 2 \mathrm{Y} 1$ and $\mathrm{P} 2 \mathrm{X} 1$, which collaborate with platelet activation causing additional damage to the P2Y12R effects. P2Y1R is generally distributed in several tissues, such as blood vessels and cells, smooth muscle, neural tissue, heart, and reproductive organs. Hence, inhibition of this receptor could result in the interference of other physiological pathways. However, in an in vivo study, prolonged bleeding time was found triggering the preservation of hemostasis. A result similar to this was demonstrated in research with the P2X1R. Both receptors, therefore, are potential therapeutic targets to be researched in the context of anti-platelet drugs [40, 42, 46]. Figure 2 illustrates the possible purinergic pathways addressed throughout the previous topics.

\section{Therapeutic possibilities in COVID-19}

The CD39 and CD73 increased activity contribute, respectively, to the reduction of ATP levels and the increase in the amount of adenosine in the site of infection. In the context of COVID-19, the modulation of these two enzymes could generate an anti-inflammatory environment by reducing the levels of ATP and by increasing the levels of adenosine, which is an immunosuppressive molecule [11]. The reduction in ATP levels would decrease chemotaxis of inflammatory cells and the effects of purinergic signaling on ATP and ADP-activated receptors: mitigating the release of cytokines (IL-1 $\beta$ ) and the activation of the inflammasome NLRP3 by P2X7R, release of cytokines (IL-1 $\beta$, IL - 6 and TNF- $\alpha$ ) [18] and damage to the pulmonary epithelium by P2Y6R [29], and platelet aggregation by P2Y12, P2Y1 and P2X1 [40-42]. In research by Ahmadi et al. [43], which was a control group study, performed an analysis of CD39 and CD73 expression pattern on $\mathrm{CD} 4^{+} \mathrm{T}, \mathrm{CD} 8^{+} \mathrm{T}$, natural killer $\mathrm{T}$, and natural killer cells of COVID-19 using flow cytometry panel; the results were a correlation between the absence of CD73 from $\mathrm{CD}^{+} \mathrm{T}$ cells and NKT and more capable of secreting granzyme $\mathrm{B}$, perforin, TNF- $\alpha$, and interferon- $\gamma(\mathrm{IFN}-\gamma)$ regardless of the disease status. But this effect was stronger in lymphocytes obtained from COVID-19 patients compared to those obtained from healthy participants [43]. In an in vitro study by Yang et al. [47], it has been shown that overexpressed CD39 negatively regulates inflammasome
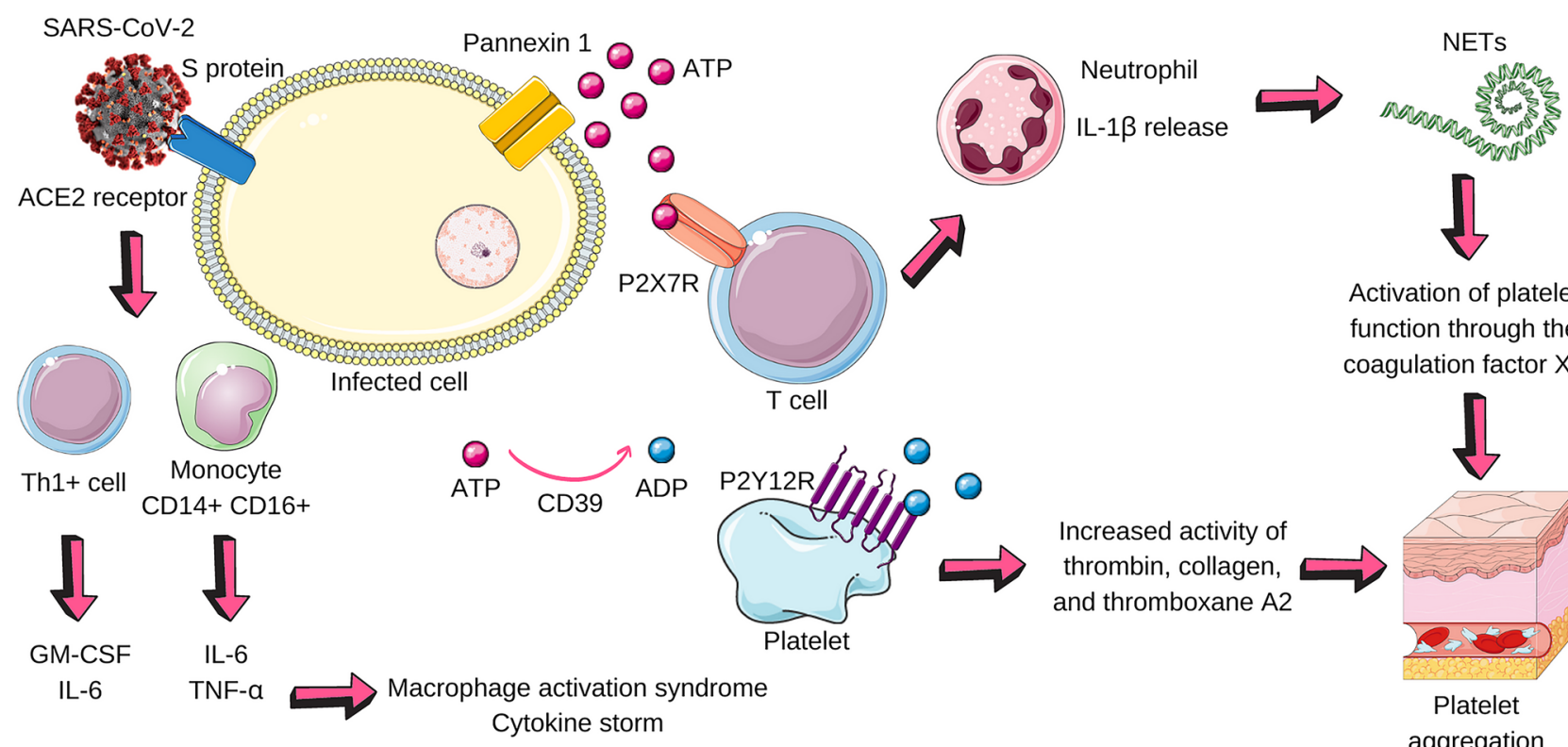

Activation of platelet function through the coagulation factor XII Cytokine storm

Fig. 2 Possible purinergic pathways in COVID-19. SARS-CoV-2 infects the cell (pneumocyte, for example) from the interaction of its surface protein with the ACE2 receptor present on the cell surface. This link activates Th1+cells, which release GM-CSF and IL-6, and monocytes, which secrete IL-6 and TNF-alpha. The microenvironment becomes pro-inflammatory and two important phenomena happen: macrophage activation syndrome (MAS) and the cytokine storm. The infected cell, in a state of stress, releases ATP through pannexin 1. ATP binds to the P2X7 receptor that promotes the release of IL- $1 \beta$ by neutrophils. CD39 dephosphorylates ATP in ADP, which acts on the P2Y12 receptor on platelets and has the following effects: to increase the activity of thrombin, collagen, and thromboxane A2. In addition to these factors that contribute to platelet aggregation, neutrophils release NETs that activate platelet factor XII. The result is platelet aggregation, excessive release of cytokines, and an inflammatory microenvironment. SARS-CoV-2 Severe Acute Respiratory Syndrome Coronavirus 2, ACE2 angiotensin-converting enzyme 2, $G M-C S F$ macrophage colony-stimulating factor, $I L-6$ interleukin-6, $T N F$-alpha tumor necrosis factor alpha, ATP adenosine triphosphate, $I L-1 B$ Interleukin 1 beta, CD39 Cluster of Differentiation 39, ADP adenosine diphosphate, NETs neutrophil extracellular traps 

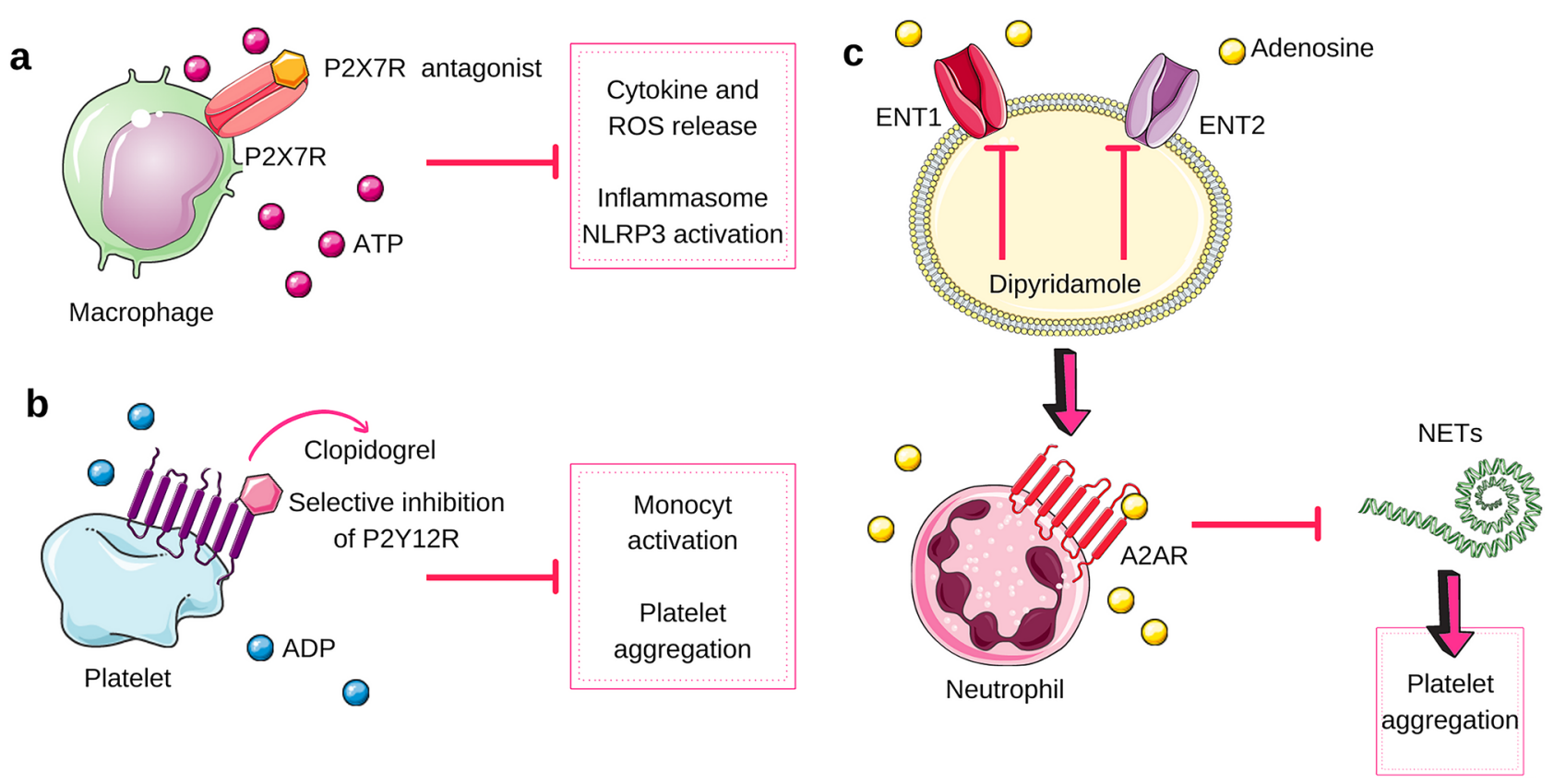

Fig. 3 Therapeutic possibilities in COVID-19. In "a," we explored the possible effects of the P2X7R antagonist (for example, AZD9056) $[54,55]$, which would have the result of inhibiting the release of cytokines and reactive oxygen species (ROS), and the activation of the inflammasome NLRP3. In "b," there is a possible outcome of clopidogrel, a selective P2Y12R inhibitor, which could reduce the monocytic activation and platelet aggregation. In "c," there is the

NLRP3 and decreases the release of ROS. There is a need for more investigation about the prognostic of this association between CD73 levels and the immune response, and about the CD39 levels in COVID-19 patients.

Regarding the increase in adenosine levels after ATP degradation, adenosine acts on $\mathrm{G}$ protein-coupled receptors $\mathrm{A} 2 \mathrm{~A}$ and $\mathrm{A} 2 \mathrm{~B}$, which increases the intracellular concentration of cyclic adenosine monophosphate (cAMP), produces anti-inflammatory effects in various tissues, and inhibits platelet activation $[48,49]$. Dipyridamole which acts on the adenosinergic pathway may be one of the therapeutic possibilities for the treatment of COVID-19, as suggested by Kanthi et al. [50]. Dipyridamole is a drug that inhibits adenosine transporters-equilibrative nucleoside transporter 1 (ENT1) and 2 (ENT2) - concomitant to the increase in extracellular levels of adenosine. This drug is used to promote coronary artery vasodilation during tests, such as echocardiography, and inhibition of platelet aggregation [51]. This inhibition is associated with an increase in cAMP [52], suppression of NET release from neutrophils through A2AR [53], in addition to causing reperfusion due to the vasodilatory effect of adenosine [52]. Therefore, the enhancement in the activity of CD39 and CD73 and the use of dipyridamole would be an important therapeutic combination possibility to the regulation of purinergic signaling in the context of COVID-19. result of using dipyridamole, which blocks the adenosine transporters ENT1 and ENT2, which generates an extracellular environment rich in adenosine. This adenosine, in turn, acts on the $\mathrm{A} 2 \mathrm{~A}$ receptors of neutrophils, preventing the formation of NETs, which no longer contributes to the formation of platelet aggregation. ENT1 equilibrative nucleoside transporter 1, ENT2 equilibrative nucleoside transporter 2, NETs neutrophil extracellular traps

However, in vitro and in vivo studies are necessary, since the other receptors covered by the review must be taken into account. Figure 3 compiles some of the main therapeutic possibilities proposed in this review.

\section{Conclusion}

In this review, we explored the possible purinergic signaling pathways and therapeutic targets in COVID-19. Cells infected by SARS-CoV-2, in a situation of cellular stress, can release ATP to the extracellular microenvironment, which activates several purinergic receptors. The use of a P2X7R antagonist would attenuate the inflammatory state, reduce the release of IL- $1 \beta$ by neutrophils, and decrease the activation of the inflammasome NLRP3. Other receptors potentially involved in the exacerbated inflammatory response would be $\mathrm{P} 2 \mathrm{Y} 2 \mathrm{R}$, its absence hinders clearance, and P2Y6R, in which inhibition in an animal model maintained its anti-microbial effects without increasing cytokines. P2X7R, P2Y2R, and P2Y6R may be related to the findings of COVID-19: infiltration of mononucleated cells in the lungs, cytokine storm, and MAS. P2Y12R, in turn, is activated by ADP and would be associated with monocyte activation and platelet aggregation. Clopidogrel inhibits this 
receptor and, therefore, represents a possibility for cases of diffuse coagulation in patients with COVID-19. There is a need for further in vivo studies on the impact of P2X1R and $\mathrm{P} 2 \mathrm{Y} 1 \mathrm{R}$ in the context of thrombosis. In addition to purinergic receptors, inducing increased CD39 and CD73 activity would increase ATP dephosphorylation in adenosine, reducing the effects mentioned in $\mathrm{P} 2 \mathrm{X}$ and $\mathrm{P} 2 \mathrm{Y}$ receptors, but also limiting the immune response. Another attractive opportunity is the inhibition of the ENT1 and ENT2 adenosine transporters by dipyridamole, which would enhance the levels of extracellular adenosine. However, it must be investigated whether the effects on $\mathrm{A} 2 \mathrm{~A}$ and $\mathrm{A} 2 \mathrm{~B}$ receptors would continue. Therefore, the purinergic system represents an important area of study to be covered in research on pathophysiology and treatment in COVID-19, given the numerous correlations between purinergic system, immunology, and coagulation.

Author contributions A.M.C. conceived of the presented idea. M.L.M.F and M.D.M.L. developed the theory and create the figures. M.R.C. encouraged the team to investigate the link between platelets and coagulation and supervised the findings of this work. A.M.C and M.R.C revised the final work.

Funding No funding was received to assist with the preparation of this manuscript.

Data availability Not applicable.

Code availability Not applicable.

\section{Declarations}

Conflict of interest The authors declare that they have no conflicts of interest.

Ethical approval Not applicable.

Consent to participate Not applicable.

Consent for publication Not applicable.

\section{References}

1. Burstock G, Knight GE (2018) The potential of P2X7 receptors as a therapeutic target, including inflammation and tumour progression. Purinergic Signal 14:1-18. https://doi.org/10.1007/ s11302-017-9593-0

2. Pfaffenzeller MS, Franciosi MLM, Cardoso AM (2020) Purinergic signaling and tumor microenvironment in cervical cancer. Purinergic Signal 16:123-135. https://doi.org/10.1007/ s11302-020-09693-3

3. World Health Organization (2020) WHO Coronavirus Disease (COVID-19) Dashboard. https://covid19.who.int/. Accessed 24 Feb 2021
4. Berlin DA, Gulick RM, Martinez FJ (2020) Severe COVID-19. N Engl J Med. https://doi.org/10.1056/NEJMcp2009575

5. Merad M, Martin JC (2020) Pathological inflammation in patients with COVID-19: a key role for monocytes and macrophages. Nat Rev Immunol 20:355-362. https://doi.org/10. 1038/s41577-020-0331-4

6. Di Virgilio F, Adinolfi E (2017) Extracellular purines, purinergic receptors and tumor growth. Oncogene 36:293-303. https:// doi.org/10.1038/onc.2016.206

7. Ledderose C, Bao Y, Kondo Y et al (2016) Purinergic signaling and the immune response in sepsis: a review. Clin Ther 38:1054-1065. https://doi.org/10.1016/j.clinthera.2016.04.002

8. Antonioli L, Blandizzi C, Pacher P, Haskó G (2019) The purinergic system as a pharmacological target for the treatment of immune-mediated inflammatory diseases. Pharmacol Rev 71:345-382. https://doi.org/10.1124/pr.117.014878

9. Della Latta V, Cabiati M, Rocchiccioli S et al (2013) The role of the adenosinergic system in lung fibrosis. Pharmacol Res 76:182-189. https://doi.org/10.1016/j.phrs.2013.08.004

10. Burnstock G, Boeynaems JM (2014) Purinergic signalling and immune cells. Purinergic Signal 10:529-564. https://doi.org/10. 1007/s11302-014-9427-2

11. Cekic C, Linden J (2016) Purinergic regulation of the immune system. Nat Rev Immunol 16:177-192. https://doi.org/10.1038/ nri.2016.4

12. Ackermann M, Verleden SE, Kuehnel M et al (2020) Pulmonary vascular endothelialitis, thrombosis, and angiogenesis in Covid19. N Engl J Med 383:120-128. https://doi.org/10.1056/NEJMo a2015432

13. Song F, Shi N, Shan F et al (2020) Emerging 2019 novel coronavirus (2019-nCoV) pneumonia. Radiology 295:210-217. https://doi.org/10.1148/radiol.2020200274

14. Polak SB, Gool IC, Cohen D et al (2020) A systematic review of pathological findings in COVID-19: a pathophysiological timeline and possible mechanisms of disease progression. Mod Pathol 33:2128-2138. https://doi.org/10.1038/ s41379-020-0603-3

15. Paces J, Strizova Z, Smrz D, Cerny J (2020) COVID-19 and the immune system. Physiol Res 69:379-388. https://doi.org/10. 33549/physiolres.934492

16. Tay MZ, Poh CM, Rénia L et al (2020) The trinity of COVID19: immunity, inflammation and intervention. Nat Rev Immunol 20:363-374. https://doi.org/10.1038/s41577-020-0311-8

17. Hu B, Huang S, Yin L (2020) The cytokine storm and COVID-19. J Med Virol. https://doi.org/10.1002/jmv.26232

18. Li G, Fan Y, Lai Y et al (2020) Coronavirus infections and immune responses. J Med Virol 92:424-432. https://doi.org/10. $1002 / j m v .25685$

19. Li S, Jiang L, Li X et al (2020) Clinical and pathological investigation of patients with severe COVID-19. JCI Insight 5:1-13. https://doi.org/10.1172/jci.insight.138070

20. Di Virgilio F, Tang Y, Sarti AC, Rossato M (2020) A rationale for targeting the $\mathrm{P} 2 \mathrm{X} 7$ receptor in Coronavirus disease 19. Br J Pharmacol 177:4990-4994. https://doi.org/10.1111/bph.15138

21. Abouelkhair MA (2020) Targeting adenosinergic pathway and adenosine A2A receptor signaling for the treatment of COVID19: a hypothesis. Med Hypotheses 144:110012. https://doi.org/ 10.1016/j.mehy.2020.110012

22. Antonioli L, Fornai M, Blandizzi C et al (2019) Adenosine signaling and the immune system: when a lot could be too much. Immunol Lett 205:9-15. https://doi.org/10.1016/j.imlet.2018.04. 006

23. Zhou Y, Mohsenin A, Morschl E et al (2009) Enhanced airway inflammation and remodeling in adenosine deaminase-deficient mice lacking the A2B adenosine receptor. J Immunol 182:80378046. https://doi.org/10.4049/jimmunol.0900515 
24. Fluentes E, Pereira J, Mezzano D et al (2014) Inhibition of platelet activation and thrombus formation by adenosine and inosine: studies on their relative contribution and molecular modeling. PLoS One 9(11):e112741. https://doi.org/10.1371/journal.pone.01127 41

25. Vliet A, Bove PF (2011) Purinergic signaling in wound healing and airway remodeling. Purinergic regulation of respiratory diseases. Springer, Dordrecht, pp 139-157

26. Olotu C, Lehmensiek F, Koch B et al (2019) Streptococcus pneumoniae inhibits purinergic signaling and promotes purinergic receptor P2Y2 internalization in alveolar epithelial cells. J Biol Chem 294:12795-12806. https://doi.org/10.1074/jbc.RA118. 007236

27. Karmakar M, Katnelson MA, Dubyak GR, Pearlman E (2016) Neutrophil P2X7 receptors mediate NLRP3 inflammasomedependent IL-1b secretion in response to ATP. Nat Commun 7:10555. https://doi.org/10.1038/ncomms 10555

28. Guo YR, Cao QD, Hong ZS et al (2020) The origin, transmission and clinical therapies on coronavirus disease 2019 (COVID-19) outbreak - an update on the status. Mil Med Res 7:11. https://doi. org/10.1186/s40779-020-00240-0

29. Cicko S, Köhler TC, Ayata CK et al (2018) Extracellular ATP is a danger signal activating P2X7 receptor in a LPS mediated inflammation (ARDS/ALI). Oncotarget 9:30635-30648. https://doi.org/ 10.18632/oncotarget.25761

30. Vanderstocken G, Paar EV, Robaye B et al (2012) Protective role of $\mathrm{P} 2 \mathrm{Y} 2$ receptor against lung infection induced by pneumonia virus of mice. PLoS One 7(11):e50385. https://doi.org/10.1371/ journal.pone. 0050385

31. Zheng J, Huang Y, Islam D et al (2018) Dual effects of human neutrophil peptides in a mouse model of pneumonia and ventilator-induced lung injury. Respir Res 19:190. https://doi.org/10. 1186/s12931-018-0869-x

32. Carter C, Osborn M, Agagah G et al (2020) COVID-19 disease: invasive ventilation. Clinics in Integrated Care 1:100004. https:// doi.org/10.1016/j.intcar.2020.100004

33. Póvoa HCC, Chianca GC, Iorio NLPP (2020) COVID-19: an alert to ventilator-associated bacterial pneumonia. Infect Dis Ther 9:417-420. https://doi.org/10.1007/s40121-020-00306-5

34. Connors JM, Levy JH (2020) COVID-19 and its implications for thrombosis and anticoagulation. Blood 135:2033-2040. https:// doi.org/10.1182/blood.2020006000

35. Fletcher-Sandersjöö A, Bellander BM (2020) Is COVID-19 associated thrombosis caused by overactivation of the complement cascade? A literature review. Thromb Res 194:36-41. https://doi. org/10.1016/j.thromres.2020.06.027

36. Cui S, Chen S, Li X et al (2020) Prevalence of venous thromboembolism in patients with severe novel coronavirus pneumonia. J Thromb Haemost 18:1421-1424. https://doi.org/10.1111/jth. 14830

37. Klok FA, Kruip MJHA, Van der Meer NJM et al (2020) Incidence of thrombotic complications in critically ill ICU patients with COVID-19. Thromb Res 191:145-147. https://doi.org/10.1016/j. thromres.2020.04.013

38. Colling ME, Kanthi Y (2020) COVID-19-associated coagulopathy: an exploration of mechanisms. Vasc Med 25:471-478. https:// doi.org/10.1177/1358863X20932640

39. Terpos E, Ntanasis-Stathopoulos EI et al (2020) Hematological findings and complications of COVID-19. Am J Hematol 95:834847. https://doi.org/10.1002/ajh.25829

40. Covarrubias R, Chepurko E, Reynolds A et al (2016) Role of the CD39/CD73 purinergic pathway in modulating arterial thrombosis in mice. Arterioscler Thromb Vasc Biol 36:1809-1820. https:// doi.org/10.1161/ATVBAHA.116.307374
41. Anyanwu AC, Kanthi Y, Fukase K et al (2019) Tuning the thrombo-inflammatory response to venous flow interruption by the ectonucleotidase CD39. Arterioscler Thromb Vasc Biol 39:118-129. https://doi.org/10.1161/ATVBAHA.119.312407

42. Hechler B, Gachet C (2015) Purinergic receptors in thrombosis and inflammation. Arterioscler Thromb Vasc Biol 35:2307-2315. https://doi.org/10.1161/ATVBAHA.115.303395

43. Ahmadi P, Hartjen P, Kohsar M et al (2020) Defining the CD39/ CD73 axis in SARS-CoV-2 infection: the CD73- phenotype identifies polyfunctional cytotoxic lymphocytes. Cells 9:1750. https:// doi.org/10.3390/cells9081750

44. Zhou F, Yu T, Du R et al (2020) Clinical course and risk factors for mortality of adult inpatients with COVID-19 in Wuhan, China: a retrospective cohort study. Lancet 395:1054-1062. https://doi. org/10.1016/S0140-6736(20)30566-3

45. Thomas MR, Outteridge SN, Ajjan RA et al (2015) Platelet P2Y12 inhibitors reduce systemic inflammation and its prothrombotic effects in an experimental human model. Arterioscler Thromb Vasc Biol 35:2562-2570. https://doi.org/10.1161/ATVBAHA. 115.306528

46. Liverani E, Rico MC, Tsygankov AY et al (2016) P2Y12 receptor modulates sepsis-induced inflammation. Arterioscler Thromb Vasc Biol. https://doi.org/10.1161/ATVBAHA.116.307401

47. Yang M, Lu L, Kang Z et al (2019) Overexpressed CD39 mitigates sepsis-induced kidney epithelial cell injury via suppressing the activation of NLR family pyrin domain containing 3. Int J Mol Med 44:1707-1718. https://doi.org/10.3892/ijmm.2019.4349

48. Di Virgilio F, Vuerich M (2015) Purinergic signaling in the immune system. Auton Neurosci 191:117-123. https://doi.org/ 10.1016/j.autneu.2015.04.011

49. Johnston-Cox HA, Yang D, Ravid K (2011) Physiological implications of adenosine receptor-mediated platelet aggregation. J Cell Physiol 226:46-51. https://doi.org/10.1002/jcp.22379

50. Kanthi Y, Knight J, Zuo Y et al (2020) New (re)purpose for an old drug: purinergic modulation may extinguish the COVID-19 thrombo-inflammatory firestorm. JCI Insight. https://doi.org/10. 1172/jci.insight. 140971

51. Eltzschig HK, Sitkovsky MV, Robson SC (2012) Purinergic signaling during inflammation. N Engl J Med 367:2322-2333. https:// doi.org/10.1056/NEJMra1205750

52. Puri N, Mohey V, Singh M et al (2016) Dipyridamole attenuates ischemia reperfusion induced acute kidney injury through adenosinergic A1 and A2A receptor agonism in rats. Naunyn Schmiedebergs Arch Pharmacol 389:361-368. https://doi.org/ 10.1007/s00210-015-1206-2

53. Ali RA, Gandhi AA, Meng H et al (2019) Adenosine receptor agonism protects against NETosis and thrombosis in antiphospholipid syndrome. Nat Commun 10:1916. https://doi.org/10.1038/ s41467-019-09801-x

54. Arulkumaran N, Unwin RJ, Tam FW (2011) A potential therapeutic role for $\mathrm{P} 2 \mathrm{X} 7$ receptor $(\mathrm{P} 2 \mathrm{X} 7 \mathrm{R})$ antagonists in the treatment of inflammatory diseases. Expert Opin Investig Drugs 20:897-915. https://doi.org/10.1517/13543784.2011.578068

55. De Marchi E, Orioli E, Dal Ben D, Adinolfi E (2016) P2X7 Receptor as a Therapeutic Target. Adv Protein Chem Struct Biol 104:39-79. https://doi.org/10.1016/bs.apcsb.2015.11.004

Publisher's Note Springer Nature remains neutral with regard to jurisdictional claims in published maps and institutional affiliations. 